\title{
Genicular Nerve Pulsed Dose Radiofrequency (PDRF) Compared to Intra-Articular and Genicular Nerve PDRF in Knee Osteoarthritis Pain: A Propensity Score-Matched Analysis
}

This article was published in the following Dove Press journal:

Journal of Pain Research

\section{Matteo Luigi Giuseppe Leoni' \\ Michael E Schatman (D) ${ }^{2,3}$ Laura Demartini ${ }^{4}$ Giuliano Lo Bianco $\mathbb{1}^{5-7}$ Gaetano Terranova $\mathbb{1 D}^{8}$}

'Unit of Interventional Pain Management, Guglielmo da Saliceto Hospital, Piacenza, Italy; ${ }^{2}$ Department of Diagnostic Sciences, Tufts University School of Dental Medicine, Boston, MA, USA; ${ }^{3}$ Department of Public Health and Community Medicine, Tufts University School of Medicine, Boston, MA, USA; ${ }^{4}$ Pain Unit, Istituti Clinici Scientifici Maugeri, Pavia, Italy; ${ }^{5}$ Basildon and Thurrock University Hospital, Essex, London, Orsett Hospital, Pain Management and Neuromodulation, London, Essex, UK; ' ${ }^{6}$ RCCS Centro Regionale Oncologico Basilicata, Rionero in Vulture, Italy; ${ }^{7}$ Department of Biomedical and Biotechnological Sciences (Biometec), Università di Catania, Catania, Italy; ${ }^{8}$ Anaesthesia and Intensive Care Department, Asst Gaetano Pini, Milano, Italy
Correspondence: Matteo Luigi Giuseppe Leoni

Unit of Interventional Pain Management, G. da Saliceto Hospital, via Taverna 49, 29I2I, Piacenza, Italy

Email matteolg.leoni@gmail.com
Background: Chronic knee osteoarthritic (OA) pain is a common and debilitating complaint in elderly patients. Despite numerous pharmaceutical options, the majority of patients still experience long-term pain. Genicular nerve (GN) radiofrequency has become increasingly popular as a treatment for knee pain. This retrospective study aimed to evaluate the effects of pulse dose radiofrequency (PDRF) in patients with chronic knee OA pain.

Patients and Methods: Propensity score matching analysis was performed in a retrospective cohort of 78 patients with moderate-severe knee OA pain unresponsive to conservative treatment who underwent PDRF GN or intra-articular (IA) and PDRF GN. Pain relief was measured using the numeric rating scale (NRS), Western Ontario and McMaster Universities Osteoarthritis Index (WOMAC) and Patient Global Impression of Change (PGIC) at 3 and 6 months post-intervention.

Results: A significant reduction in NRS scores was reported at $3(p<0.001)$ and 6 months $(\mathrm{p}<0.001)$ after PDRF in both groups. NRS was lower in PDRF IA + GN than PDRF GN $(\mathrm{p}<0.0001)$. WOMAC pain was significantly reduced at 3 months in PDRF IA + GN group (baseline: $10.12 \pm 3.14,3$ months: $6.25 \pm 2.44, \mathrm{p}=0.0001$ ). WOMAC stiffness and function were improved only at 3 months in PDRF IA + GN compared to baseline $(p=0.007$ and $\mathrm{p}=0.006$, respectively). A longer period of pain relief was reported after PDRF IA $+\mathrm{GN}$ $(6.75 \pm 2.42$ months $)$ compared to PDRF GN $(4.31 \pm 2.85$ months, $\mathrm{p}<0.001)$ in association with higher PGIC scores.

Conclusion: This is the first study that compared two different PDRF techniques. PDRF GN and PDRF IA + GN were both effective in reducing pain at 3 and 6 months follow-up. However, only PDRF IA + GN was able to improve WOMAC scores at 3 months after the treatment with a longer period of efficacy compared to PDRF GN alone.

Keywords: knee pain, pulse dose radiofrequency; PDRF, radiofrequency; genicular nerve, osteoarthritis, chronic pain, WOMAC, interventional pain management

\section{Introduction}

Symptomatic knee osteoarthritis (OA) is very common among older people. ${ }^{1}$ Its prevalence in subjects over 60 years-old is $12.2 \%$ and is notably higher in women $(14.9 \%)$ than in men $(8.7 \%)$. It increases with age, obesity and mechanical stress and leads to considerable social costs. ${ }^{2}$ Common causes of OA include a disruption of homeostatic state of balanced anabolism and catabolism of the cartilage 
extracellular matrix with a shift toward a catabolic environment. This eventually leads to macroscopic hyaline cartilage degeneration and synovial overgrowth associated with inflammatory changes and bony hypertrophy (osteophyte formation). According to the OARSI guidelines, the first-line treatment of knee osteoarthritis includes nonpharmacological strategies (exercise programs, dietary weight management and education about OA) and secondly, topical or oral anti-inflammatory drugs, intraarticular hyaluronic acid and steroid injections. ${ }^{3}$ Unfortunately, these therapies have demonstrated little effect in many patients, as well as undesirable side effects. Moreover, the resulting increase in opioid prescription has likely contributed to a dramatic increase in the number of accidental falls in the elderly with increased morbidity. ${ }^{4}$ Arthroscopy or knee arthroplasty are often considered when other medical therapies fail to relieve symptoms. ${ }^{5}$

Pulsed dose radiofrequency (PDRF) is an evolution of conventional radiofrequency with less or no correlation with neural damage. ${ }^{6}$ Considerable efficacy in reducing symptoms of knee OA has been established when this technique was applied to genicular ${ }^{7}$ (GN) or intraarticular (IA) nerves. ${ }^{8,9}$ In long-standing OA, it results in consistent improvement of reported pain, swelling, and stiffness. PRF creates a neuromodulatory effect, suppressing both excitatory C-fibers activation and the spread of pain impulse at the synaptic junction, in addition to a modulatory effect on pro-inflammatory cytokines. ${ }^{10,11}$

The purpose of this retrospective study was to evaluate the effects of PDRF applied to GN or to IA + GN in patients with OA knee pain refractory to conservative treatments. Propensity score matching was used to reduce patients' selection bias and to produce two groups that were comparable in terms of demographic profile and disease characteristics.

\section{Methods}

This is a single-center clinical cohort study based on a retrospective analysis of prospectively collected data on 78 consecutive patients with moderate-severe knee OA pain unresponsive to conservative treatment who underwent PDRF. The study was conducted at the Pain Unit of ICS Maugeri Hospital, Pavia, Italy, from January 2018 to December 2018. All participants signed a written informed consent. The study was approved by the hospital's Institutional Ethics Committee and was conducted in accordance with the Declaration of Helsinki. All patients were evaluated before PDRF with a knee x-ray and physical examination to ascertain their eligibility. The diagnosis and classification of knee osteoarthritis' severity were conducted through the Kellgren-Lawrence method. ${ }^{12}$ Patients with knee pain unresponsive to conservative treatment (physiotherapy, oral analgesics) and intra-articular injection with steroids or hyaluronic acid were included in this study. Radicular pain (mainly L3-L4), pain posttotal knee replacement, rheumatoid arthritis, complex regional pain syndrome or history of intra-articular injection with steroid or hyaluronic acid within the previous 3 months were considered as exclusion criteria. All enrolled patients underwent a successful diagnostic genicular nerve block with local anesthetic (Lidocaine 1\%, $2 \mathrm{~mL}$ ) prior to PDRF. $^{13}$

\section{Patients}

Patients were assigned to PDRF GN or PDRF IA + GN according to physicians' preference and experience with the technique used.

An outcome investigation was performed in both groups at 3 and 6 months following the procedures. Pain intensity (NRS), Western Ontario and McMaster Universities Osteoarthritis Index (WOMAC) and Patient Global Impression of Change (PGIC) scores were obtained before PDRF and at each follow-up.

\section{Technique}

PDRF GN and PDRF IA + GN were performed with a $22 \mathrm{G} \times 100 \mathrm{~mm}$ needle (NeuroTherm ${ }^{\mathrm{TM}}$, Medipoint GmbH, Hamburg, Germany) with $10 \mathrm{~mm}$ active tip. Fluoroscopic confirmation of the needle position was used for PDRF IA, while both fluoroscopic and ultrasound assessment of topographical localization of superior lateral, superior medial and inferior medial genicular nerve branches were used for PDRF GN. The superior medial genicular nerve curves around the femoral shaft pass on the femoral medial epicondyle to descend $1 \mathrm{~cm}$ anterior to the adductor tubercle, where the needle was placed. The inferior medial genicular nerve is situated around the tibial medial epicondyle and was found at the tibial insertion of the medial collateral ligament. The superior lateral genicular nerve was found at the junction between the femoral shaft and the femoral lateral epicondyle. Nerve localization was confirmed with stimulation at $\leq 0.4 \mathrm{mV}, 50 \mathrm{~Hz}$. PDRF was performed using the following parameters: 1200 pulses at high voltage (45 V), with $20 \mathrm{~ms}$ duration followed by $480 \mathrm{~ms}$ silent phases. 


\section{Statistical Analyses}

Propensity score matching analysis was used with the nearest matching algorithm $(1: 1$ ratio, caliper value $=0.2)$. Patients' characteristics selected for the matching analysis were: age, body mass index (BMI), basal NRS, basal WOMAC total score and Kellgren-Laurence knee OA grade.

Comparisons between the two groups were carried out with a Mann-Whitney test ( $U$-test), after evaluating the normality of the distribution with the Kolmogorov-Smirnov test.

The differences of the quantitative variables at different time points were analyzed with ANOVA for repeated measures with Bonferroni adjustment for multiple comparisons. A p-value $<0.05$ was considered statistically significant. Data are expressed as mean \pm SD. STATA V.15 (STATA Corp., Texas, USA) was used for the analyses.

\section{Results}

112 patients with knee OA pain were screened and 34 did not meet the inclusion criteria. The reason for exclusion included: histories of steroid or hyaluronic acid intraarticular injections within 3 months (13 patients), L3-L4 radiculopathy ( 7 patients), pain following total knee replacement (7 patients), rheumatoid arthritis (6 patients) and complex regional pain syndrome type 1 (1 patient). Therefore, 78 patients met the inclusion criteria. Propensity score matching analysis resulted in 27 PDRF GN and 27 PDRF IA + GN matched cases, which comprise the study population used for comparisons. Age, BMI, basal NRS, basal WOMAC total score and radiographic disease severity were compared within the matched PDRF GN and PDRF IA + GN groups to evaluate the accuracy of the matching process. No statistically significant difference was observed in any of the above parameters, as demonstrated in Table 1. Female patients accounted for the majority of the sample (72\%).

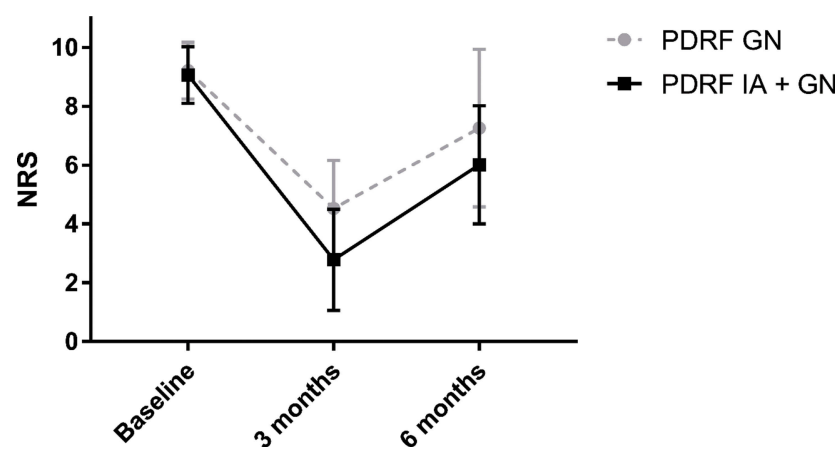

Figure I Pain intensity measured with a numerical rating scale (NRS) at 3 and 6 months after PDRF GN or PDRF IA + GN. The significant reduction observed at 3 months $(p<0.000 I)$ was maintained, although weakened, at 6 months $(p=0.006)$.
Table I Population Variables. Comparison of Clinical Characteristic Between the PDRF GN and PDRF IA + GN Groups after the matching process

\begin{tabular}{|l|c|c|l|}
\hline $\begin{array}{l}\text { Population } \\
\text { Variables }\end{array}$ & $\begin{array}{c}\text { PDRF GN } \\
(\mathbf{n = 2 7 )}\end{array}$ & $\begin{array}{c}\text { PDRF IA + GN } \\
(\mathbf{n = 2 7 )}\end{array}$ & $\mathbf{P}$ \\
\hline Age & $75.3 \pm 7.9$ & $76.8 \pm 9.45$ & 0.44 \\
BMI & $27.1 \pm 5.6$ & $26.5 \pm 4.9$ & 0.61 \\
NRS basal & $9.2 \pm 0.97$ & $9.1 \pm 0.96$ & 0.73 \\
WOMAC total score & $71.8 \pm 14.6$ & $72.27 \pm 13.5$ & 0.91 \\
(basal) & & & \\
\hline Radiographic knee OA & & & \\
severity & & & \\
$\quad$ Grade 2 & 8 & 7 & 0.76 \\
Grade 3 & 14 & 13 & 0.59 \\
Grade 4 & 5 & 7 & $0.5 \mathrm{I}$ \\
\hline
\end{tabular}

A significant reduction in NRS score was reported in both groups at 3 months $(\mathrm{p}<0.001)$ and 6 months $(\mathrm{p}<0.001)$ compared to the baseline (PDRF GN: baseline NRS 9.2 $\pm 0.97,3$ months NRS 4.5 $\pm 1.63,6$ months NRS 7.2 \pm 2.68 ; PDRF IA + GN: baseline NRS 9.1 $\pm 0.96,3$ months NRS $2.7 \pm 1.71,6$ months NRS 6 \pm 2.0 ). At 3 months, the PDRF IA + GN group's NRS was significantly lower than PDRF GN group's NRS $(\mathrm{p}<0.0001)$. At 6 months, the same results were confirmed although weakened $(\mathrm{p}=0.006)$ (Figure 1).

No change in WOMAC pain was found in the PDRF GN group at 3 months $(p=0.40)$ and 6 months after the treatment $(\mathrm{p}=0.52)$. Otherwise, WOMAC pain was significantly reduced at 3 months in the PDRF IA + GN group (WOMAC pain baseline 10.12 \pm 3.14 , WOMAC pain three months $6.25 \pm 2.44, \mathrm{p}=0.0001$ ). Moreover, WOMAC pain was reduced in PDRF IA + GN group compared to PDRF $\mathrm{GN}$ at 3 months ( $\mathrm{p}=0.005$ ) (Figure 2).

WOMAC stiffness was improved only at 3 months in the PDRF IA + GN group compared to baseline (WOMAC stiffness baseline $4.96 \pm 1.83$, WOMAC stiffness three months $3.71 \pm 1.23, \mathrm{p}=0.007$ ) and no changes were found at different time points for PDRF GN. WOMAC stiffness was reduced in PDRF IA + GN group compared to PDRF GN only at 3 months $(\mathrm{p}=0.02)$ (Figure 2$)$.

WOMAC function was improved only in the PDRF IA + GN group at 3 months after the treatment (WOMAC function baseline 55.08 \pm 14.52 , WOMAC function three months $41.04 \pm 11.70, p=0.0006)$. No differences in WOMAC function were found between the two treatments at different time points (Figure 2). The WOMAC total score was significantly improved only in the PDRF IA + GN group at 3 months $(\mathrm{p}<0.001)$. 

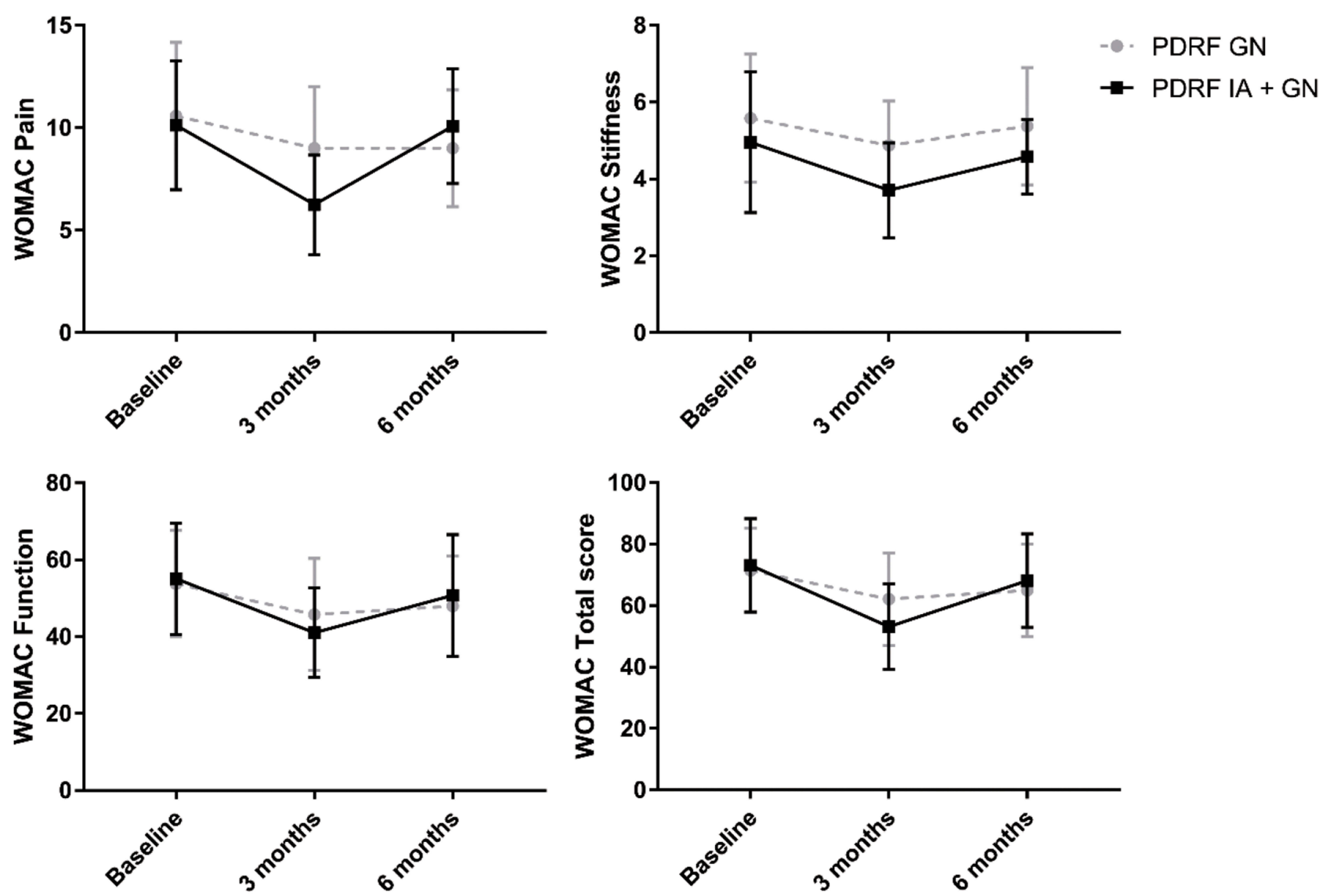

Figure 2 Western Ontario and McMaster Universities Osteoarthritis Index (WOMAC)showed an improvement in pain, stiffness, and function at 3 months post-procedure in the PDRF IA + GN group. WOMAC pain and WOMAC stiffness were significantly improved in the PDRF IA + GN group compared to PDRF GN (see the text). No differences were found for WOMAC function. The WOMAC total score was significantly improved only in the PDRF IA + GN group at 3 months.

Patients reported a longer period of pain relief following PDRF IA + GN (6.75 \pm 2.42 months) compared to PDRF GN $(4.31 \pm 2.85$ months, $\mathrm{p}<0.001)$. PGIC score was also better in PDRF IA + GN (PDRF GN 1.8 \pm 0.93 vs PDRF IA + GN 2.5 $\pm 0.81, \mathrm{p}=0.005)$.

Ten patients suffering from knee OA pain mainly located at the femoropatellar joint and confirmed by radiological degenerative findings did not experience any significant pain relief following PDRF. No patients developed significant complications after PDRF during the follow-up period.

\section{Discussion}

This study compares the efficacy of PDRF GN versus PDRF IA + GN in patients with moderate-severe knee OA pain unresponsive to conservative treatment. Pain intensity and knee function were analyzed at 1 and 3 months postprocedure follow-up.

PDRF has no neurodestructive effects since it maintains tissue temperature under $42^{\circ} \mathrm{C}$, which is below the irreversible tissue damage threshold. ${ }^{14}$ However, histological studies demonstrated ultrastructural changes in the $\mathrm{C}$ and A $\delta$ nociceptive fibers after PDRF. ${ }^{15,16}$

On the contrary, intra-articular application of PDRF reduces the response of $\mathrm{C}$ fiber along with a reduction of the pro-inflammatory cytokines such as interleukin- $1 \beta$ and interleukin- $6 .{ }^{17}$ As suggested by Sluijter et al, the therapeutic effect of PDRF IA is related to the action of electric fields on immune cells. In fact, in joints with an "open" geometry such as the knee the deflection of the current by bony surfaces forcing the electric filed to remain inside the joint space is very limited. ${ }^{11}$

Serdar and colleagues investigated the use of ultrasound-guided PDRF GN and demonstrated a 50\% pain reduction after 12 weeks with an improvement of WOMAC scores. ${ }^{7}$ Similarly, Masala et al investigated the effectiveness of intra-articular knee PDRF in patients with chronic knee pain unresponsive to conservative therapies and reported an improvement in pain intensity and WOMAC up to 1 year after the procedure. ${ }^{8}$ El-Hakeim 
et al recently reported in a randomized controlled trial that genicular nerve radiofrequency is not only effective for pain reduction and knee function improvement, but is superior to conventional medical therapy. ${ }^{18}$

Our data addressed the results of two different PDRF procedures at 3 and 6 months follow-up. PDRF GN and PDRF IA + GN were both able to significantly reduce NRS scores at 3 months $(\mathrm{p}<0.001)$ and 6 months $(\mathrm{p}<0.001)$ compared to the baseline. WOMAC pain, WOMAC function, WOMAC stiffness and WOMAC total score were significantly improved only in the PDRF IA + GN group at 3 months following the treatment. Moreover, the PDRF IA + GN group demonstrated a better outcome in terms of pain reduction and WOMAC at 3 months compared to the PDRF GN group. The PDRF IA + GN group had a longer period of pain relief and better patient global impression of change at 6 months following treatment compared to the group receiving PDRF GN alone.

This is the first study that describes the association of the two techniques, although further investigations through randomized controlled trials are needed to confirm our findings.

A possible explanation for the increased efficacy of PDRF IA + GN in our patients could be related to the increased electromagnetic field that can act on capsular and genicular nerves, both of which are involved in knee pain nociception. ${ }^{19}$ Moreover, the total amount of energy delivered to knees' anatomical structures with PDRF IA + GN is greater than PDRF GN as a monotherapy. As recently published, a bipolar PDRF IA seems to be more advantageous in reducing chronic knee pain and functional recovery compared with the unipolar approach. ${ }^{9}$ Eyigor et al reported knee pain reduction but unmodified functional affects (no change in WOMAC, $20 \mathrm{~m}$ walking and 6-min walk test) after PDRF IA. ${ }^{20}$ Karaman and his group confirmed the global effect of knee pain reduction after PDRF IA but they did not investigate the impact of this technique on knee function. ${ }^{21}$ An improvement in knee's function after PDRF associated with viscosupplementation was reported by Filippiadis et al. ${ }^{17}$ Unfortunately, the authors analyzed the knee function and mobility only with patients' verbal reports without standardized scales. Based on these findings, it is reasonable to hypothesize that PDRF IA can reduce pain but it is probably not effective alone in improving knee function.

PDRF techniques are effective treatments for knee OA pain non-responsive to conservative measures. Nevertheless, a standardization of the technique is needed to allow definitive acceptance of PDRF as a treatment available for knee OA. Due to the complex origin of knee joint pain, it is generally recommended to perform a nerve block with local anesthetics before PDRF as confirmed by McCormick et al. ${ }^{22}$ Surprisingly, a recently published study by the same authors showed that the anesthetic block did not improve the patient selection and the rate of radiofrequency treatment success. ${ }^{23}$

Although a propensity score matching analysis could be considered comparable to a randomized trial, ${ }^{24}$ this study still has limitations. The first is the absence of true randomization between the two PDRF treatment groups. In our study, physicians were permitted to treat patients with PDRF GN or PDRF IA + GN at their own desire. This can cause a possible degree of investigator bias. However, the utilization of a propensity matching technique was useful to reduce the likelihood of such bias. Hence, if we consider Table 1, we can see that the two matched groups presented the same preoperative characteristics. Second, our Center did not program any short term follow-up (at 4 and 8 weeks) for these patients. Consequently, data on the immediate posttreatment period are lacking, and the retrospective nature of this study does not allow us to obtain the data needed to ascertain immediate post-treatment effects. Another possible limitation of this study is that the patients' outcomes have been evaluated only according to the WOMAC scale, the NRS and the PGIC scores. As reported in the literature, additional measures such as the Chair Stand test or the time $20 \mathrm{~m}$ walk test could have been used to even better characterize the functional improvement of these patients. ${ }^{25}$

This study is retrospective in nature. Accordingly, a future prospective investigation will be required to substantiate the superiority of one technique compared to the other. Nonetheless, the differences identified between the two groups seem to indicate an additive effect of PDRF IA+ GN compared to PDRF GN.

It is noteworthy that patients from both groups who experienced no benefit after PDRF treatment originally manifested pain mainly concentrated at the femoropatellar joint, and simultaneously demonstrated radiographic indicators of patellofemoral joint OA (osteophyte formation, joint space narrowing, subluxation or dislocation, bone destruction). Additional investigations are needed to better understand the ideal PDRF treatment for femoropatellar OA pain.

\section{Conclusions}

In the current study, PDRF GN and PDRF IA + GN were both able to significantly reduce the NRS score at 3 months and 6 months compared to baseline. WOMAC pain, 
WOMAC function, and WOMAC stiffness were significantly improved only in the PDRF IA + GN group at 3 months following the treatment. The PDRF IA + GN group demonstrated a significant WOMAC improvement compared to the PDRF GN group. PDRF IA + GN had a longer period of efficacy compared to PDRF GN and a better PGIC. A prospective analysis is still needed to improve our understanding of the relative benefits of the two PDRF techniques.

\section{Disclosure}

Dr Michael E Schatman reports consultancy for Kaleo Pharma, Salix Pharmaceuticals, and Quest Diagnostics, outside the submitted work. Drs Laura Demartini reports personal fees from Boston Scientific, personal fees from Abbott, personal fees from Grunenthal Italia, outside the submitted work. The authors report no other conflicts of interest in this work.

\section{References}

1. Quintana JM, Arostegui I, Escobar A, Azkarate J, Goenaga JI, Lafuente I. Prevalence of knee and hip osteoarthritis and the appropriateness of joint replacement in an older population. Arch Intern Med. 2008;168(14):1576-1584. doi:10.1001/archinte.168.14.1576

2. Guillemin F, Rat AC, Mazieres B, et al. 3000 osteoarthritis group. prevalence of symptomatic hip and knee osteoarthritis: a two-phase population-based survey. Osteoarthritis Cartilage. 2011;19(11):13 14-1322. doi:10.1016/j.joca.2011.08.004

3. Bannuru RR, Osani MC, Vaysbrot EE, et al. OARSI guidelines for the non-surgical management of knee, hip, and polyarticular osteoarthritis. Osteoarthritis Cartilage. 2019;27(11):1578-1589. doi:10.1016/j. joca.2019.06.011

4. Rolita L, Spegman A, Tang X, Cronstein BN. Greater number of narcotic analgesic prescriptions for osteoarthritis is associated with falls and fractures in elderly adults. J Am Geriatr Soc. 2013;61 (3):335-340. doi:10.1111/jgs.12148

5. Gademan MG, Hofstede SN, Vliet Vlieland TP, et al. Indication criteria for total hip or knee arthroplasty in osteoarthritis: a state-ofthe-science overview. BMC Musculoskelet Disord. 2016;17:463. doi:10.1186/s12891-016-1325-z

6. Li DY, Meng L, Ji N, Luo F. Effect of pulsed radiofrequency on rat sciatic nerve chronic constriction injury: a preliminary study. Chin Med J. 2015;128(4):540-544. doi:10.4103/0366-6999.151113

7. Kesikburun S, Yaşar E, Uran A, Adigüzel E, Yilmaz B. Ultrasoundguided genicular nerve pulsed radiofrequency treatment for painful knee osteoarthritis: a preliminary report. Pain Physician. 2016;19: E751-E759.

8. Masala S, Fiori R, Raguso M, Morini M, Calabria E, Simonetti G. Pulsedose radiofrequency for knee osteoarthritis. Cardiovasc Intervent Radiol. 2014;37(2):482-487. doi:10.1007/s00270-013-0694-z

9. Gulec E, Ozbek H, Pektas S, Isik G. Bipolar versus unipolar intraarticular pulsed radiofrequency thermocoagulation in chronic knee pain treatment: a prospective randomized trial. Pain Physician. 2017;20 (3):197-206. doi:10.36076/ppj.2017.206
10. Das B, Conroy M, Moore D, Lysaght J, McCrory C. Human dorsal root ganglion pulsed radiofrequency treatment modulates cerebrospinal fluid lymphocytes and neuroinflammatory markers in chronic radicular pain. Brain Behav Immun. 2018;70:157-165. doi:10.1016/ j.bbi.2018.02.010.

11. Sluijter ME, Teixeira A, Serra V, Balogh S, Schianchi P. Intraarticular application of pulsed radiofrequency for atherogenic pain: report of six cases. Pain Pract. 2008;8:57e61. doi:10.1111/j.15332500.2007.00172.x

12. Kellgren JH, Lawrence JS. Radiological assessment of osteo-arthrosis. Ann Rheum Dis. 1957;16:494-502. doi:10.1136/ard.16.4.494

13. Yasar E, Kesikburun S, Kılıç C, Güzelküçük Ü, Yazar F, Tan AK. Accuracy of ultrasound-guided genicular nerve block: a cadaveric study. Pain Physician. 2015;18(5):E899-904.

14. Erdine S, Bilir A, Cosman ER, Cosman Jr. ER Jr. Ultrastructural changes in axons following exposure to pulsed radiofrequency fields. Pain Pract. 2009;9(6):407-417. doi:10.1111/j.1533-2500.2009.00317.x

15. Tun K, Cemil B, Gurcay AG, et al. Ultrastructural evaluation of pulsed radiofrequency and conventional radiofrequency lesions in rat sciatic nerve. Surg Neurol. 2009;72(5):496-500. doi:10.1016/j. surneu.2008.11.016

16. Protasoni M, Reguzzoni M, Sangiorgi S, et al. Pulsed radiofrequency effects on the lumbar ganglion of the rat dorsal root: a morphological light and transmission electron microscopy study at acute stage. Eur Spine J. 2009;18:473-478. doi:10.1007/s00586-008-0870-z

17. Filippiadis D, Velonakis G, Mazioti A, et al. Intra-articular application of pulsed radiofrequency combined with viscosupplementation for improvement of knee osteoarthritis symptoms: a single centre prospective study. Int J Hyperthermia. 2018;34(8):1265-1269. doi:10.1080/02656736.2017.1409910

18. El-Hakeim EH, Elawamy A, Kamel EZ, et al. fluoroscopic guided radiofrequency of genicular nerves for pain alleviation in chronic knee osteoarthritis: a single-blind randomized controlled trial. Pain Physician. 2018;21(2):169-177.

19. Tran J, Peng PWH, Lam K, Baig E, Agur AMR, Gofeld M. Anatomical study of the innervation of anterior knee joint capsule: implication for image-guided intervention. Reg Anesth Pain Med. 2018;43(4):407-414. doi:10.1097/AAP.0000000000000778

20. Eyigor C, Eyigor S, Akdeniz S, Uyar M. Effects of intra articular application of pulsed radiofrequency on pain, functioning and quality of life in patients with advanced knee osteoarthritis. J Back Musculoskelet Rehabil. 2015;28(1):129-134. doi:10.3233/BMR-140500

21. Karaman H, Tüfek A, GÖ K, et al. Intra-articularly applied pulsed radiofrequency can reduce chronic knee pain in patients with osteoarthritis. J Chin Med Assoc. 2011;74(8):336-340. doi:10.1016/ j.jcma.2011.06.004.

22. McCormick ZL, Korn M, Reddy R, et al. Cooled radiofrequency ablation of the genicular nerves for chronic pain due to knee osteoarthritis: six-month outcomes. Pain Med. 2017;18(9):1631-1641. doi:10.1093/pm/pnx069

23. McCormick ZL, Reddy R, Korn M, et al. A prospective randomized trial of prognostic genicular nerve blocks to determine the predictive value for the outcome of cooled radiofrequency ablation for chronic knee pain due to osteoarthritis. Pain Med. 2018;19(8):1628-1638. doi: $10.1093 / \mathrm{pm} / \mathrm{pn} \times 286$

24. Austin PC. An introduction to propensity score methods for reducing the effects of confounding in observational studies. Multivariate Behav Res. 2011;46(3):399-424. doi:10.1080/00273171.2011.568786.

25. Dobson F, Hinman RS, Roos EM, et al. OARSI recommended performance-based tests to assess physical function in people diagnosed with hip or knee osteoarthritis. Osteoarthritis Cartilage. 2013;21(8):1042-1052. doi:10.1016/j.joca.2013.05.002 


\section{Publish your work in this journal}

The Journal of Pain Research is an international, peer reviewed, open access, online journal that welcomes laboratory and clinical findings in the fields of pain research and the prevention and management of pain Original research, reviews, symposium reports, hypothesis formation and commentaries are all considered for publication. The manuscript management system is completely online and includes a very quick and fair peer-review system, which is all easy to use. Visit http:// www.dovepress.com/testimonials.php to read real quotes from published authors.

Submit your manuscript here: https://www.dovepress.com/journal-of-pain-research-journal 